Revue Revue de l'histoire des religions

$$
\begin{array}{cl}
\text { de l'histoire } & \text { 3|2012 } \\
\text { des religions } & \text { Varia }
\end{array}
$$

\title{
Jean-Baptiste YON et Pierre-Louis GATIER (dir.), Choix d'inscriptions grecques et latines de la Syrie
}

Amman-Beyrouth-Damas-Alep, 2009, L'Institut français du Proche-Orient (« Guides archéologiques de l'Institut français d'archéologie du ProcheOrient », 6), 223 p., 24 cm, $30 €$, ISBN 978-2-35159-080-5.

\section{Sylvain Destephen}

\section{OpenEdition}

Journals

Édition électronique

URL : http://journals.openedition.org/rhr/7913

DOI : 10.4000/rhr.7913

ISSN : 2105-2573

Éditeur

Armand Colin

Édition imprimée

Date de publication : 1 septembre 2012

Pagination : 413-416

ISBN : 978-2200-92975-0

ISSN : 0035-1423

Référence électronique

Sylvain Destephen, « Jean-Baptiste yon et Pierre-Louis gatIER (dir.), Choix d'inscriptions grecques et

latines de la Syrie », Revue de l'histoire des religions [En ligne], 3 | 2012, mis en ligne le 04 octobre 2012, consulté le 22 septembre 2020. URL : http://journals.openedition.org/rhr/7913 ; DOI : https://doi.org/ $10.4000 /$ rhr.7913

Ce document a été généré automatiquement le 22 septembre 2020

Tous droits réservés 


\section{Jean-Baptiste YoN et Pierre-Louis GATIER (dir.), Choix d'inscriptions grecques et latines de la Syrie}

Amman-Beyrouth-Damas-Alep, 2009, L'Institut français du Proche-Orient ( Guides archéologiques de l'Institut français d'archéologie du ProcheOrient », 6), 223 p., 24 cm, $30 €$, ISBN 978-2-35159-080-5.

\section{Sylvain Destephen}

\section{RÉFÉRENCE}

Jean-Baptiste yon et Pierre-Louis GATIER (dir.), Choix d'inscriptions grecques et latines de la Syrie, Amman-Beyrouth-Damas-Alep, 2009, L'Institut français du Proche-Orient (" Guides archéologiques de l'Institut français d'archéologie du Proche-Orient », 6), 223 p., $24 \mathrm{~cm}, 30 €$, ISBN 978-2-35159-080-5.

1 Cette sélection de 64 inscriptions est le fruit du travail collectif de sept épigraphistes, tous chercheurs au CNRS, universitaires ou pensionnaire de l'Institut français du Proche-Orient : Julien Aliquot (textes $\mathrm{n}^{\text {os }} 45$ et 54 ), Jean-Claude Decourt (textes $\mathrm{n}^{\text {os }} 1,21$, $41,42,43,48,56,58$ ), Denis Feissel (textes $n^{o s} 16,18,19,20,24,25,26,27,28,52$ ), PierreLouis Gatier (textes $\mathrm{n}^{\text {os }} 10,14,17,33,39,40,50,53,57,59$ ), Jean-Paul Rey-Coquais (textes $\mathrm{n}^{\text {os }} 2,3,7,9,15,30,34,36,38,63,64$ ), Maurice Sartre (textes $\mathrm{n}^{\text {os }} 8,13,22,23,31$, $44,46,47,49,60,61,62$ ) et Jean-Baptiste Yon (textes $\mathrm{n}^{\circ \mathrm{s}} 4,5,6,11,12,29,32,35,37,51$, 55). Tous ces spécialistes de l'Orient romain collaborent au programme des Inscriptions grecques et latines de la Syrie (ensuite abrégées en IGLS), que Pierre-Louis Gatier coordonne depuis la Maison de l'Orient et de la Méditerranée à Lyon, action menée sur place et relayée en Jordanie, au Liban et en Syrie par les antennes et les personnels de l'Institut français du Proche-Orient à Amman, Beyrouth, Damas et Alep.

Une brève introduction définit l'objet et les principes de l'épigraphie comme discipline scientifique et évoque la diversité linguistique de l'Orient romain. Plutôt que de fournir 
des exemples des principales langues connues par des inscriptions, les auteurs ont privilégié le grec qui totalise 47 des 64 inscriptions réunies dans ce petit corpus. Ces témoignages épigraphiques de l'usage abondant et croissant de la langue grecque dans cette partie de l'Empire, sont complétés par 11 inscriptions latines, 4 inscriptions bilingues -3 emploient le grec et l'araméen, 1 le grec et le latin - et 2 inscriptions trilingues combinant le grec et le latin à l'araméen. Les autres langues locales, de nature indigène, comme le phénicien, l'hébreu, le safaïtique ou le syriaque ne sont pas représentées, sans doute en raison du très petit nombre de connaisseurs de ces langues fort peu enseignées au sein des universités auxquelles se destine le présent ouvrage. La répartition chronologique des inscriptions retenues privilégie les périodes les mieux représentées par la production épigraphique régionale, à savoir l'époque romaine et protobyzantine ( $c f$. la chronologie proposée p.19-21), aux dépens de la période hellénistique. Ne sont ainsi retenues que 3 inscriptions datées des $\mathrm{III}^{\mathrm{e}} \mathrm{I}^{\mathrm{er}}$ siècles avant J.C., tandis qu'on dénombre 36 inscriptions des I $^{\mathrm{er}}$-III ${ }^{\mathrm{e}}$ siècles après J.-C., 23 inscriptions des $\mathrm{IV}^{\mathrm{e}}-\mathrm{VII}{ }^{\mathrm{e}}$ siècles et 2 inscriptions médiévales rédigées en grec (textes ${ }^{\text {os }} 20$ et 64 mentionnant, l'un la fortification de la citadelle de Qal'at Sam'an en 966, l'autre la sépulture d'un moine inhumé à Gabala en 1043 ou 1089 d'après une indication chronologique incertaine offrant plusieurs interprétations possibles).

Comme l'illustre la belle carte figurant au début du recueil (p.8), les 64 inscriptions sélectionnées proviennent toutes du territoire de l'actuelle République arabe syrienne, ce qui donne sa pleine justification au titre de l'ouvrage, même si les territoires du Liban et de la Jordanie, autrefois inclus dans la Syrie antique, sont également couverts par le programme des IGLS, dont l'introduction fait le bref historique, depuis le premier corpus homonyme publié en 1870 par l'épigraphiste et homme politique William-Henry Waddington jusqu'aux travaux pionniers, mais aujourd'hui quelque peu vieillis, des Pères jésuites Louis Jalabert et René Mouterde. Pour répondre aux exigences actuelles de la recherche scientifique et bénéficier des nouvelles possibilités offertes par la photographie et la reprographie, les volumes les plus anciens sont en cours de refonte, des volumes modernisés ont paru depuis les années 1960 tandis que d'autres volumes s'annoncent.

L'ampleur des découvertes réalisées et des corrections proposées depuis la parution des premiers tomes des IGLS se manifeste dans le choix des inscriptions retenues dans le présent recueil. Sur un ensemble de 64 inscriptions, seules 19 d'entre elles ont déjà été publiées dans les différents volumes du corpus (texte $\mathrm{n}^{\circ} 1=I G L S, \mathrm{~V}, 1998 ; \mathrm{n}^{\circ} 7=\mathrm{VII}$, 4016 bis ; $\mathrm{n}^{\circ} 13=\mathrm{XVI}, 1344 ; \mathrm{n}^{\circ} 16=\mathrm{II}, 348 ; \mathrm{n}^{\circ} 18=\mathrm{IV}, 1682 ; \mathrm{n}^{\circ} 19=\mathrm{II}, 281 ; \mathrm{n}^{\circ} 21=\mathrm{V}$, $2549-2551 ; n^{\circ} 24=I I, 417 ; n^{\circ} 25=I V, 1459 ; n^{\circ} 27=I I, 618 ; n^{\circ} 28=I I, 530 ; n^{\circ} 34=V I I$, $4028 ; \mathrm{n}^{\circ} 39=\mathrm{II}, 569 ; \mathrm{n}^{\circ} 42=\mathrm{V}, 2219 ; \mathrm{n}^{\circ} 45=\mathrm{XI}, 22 ; \mathrm{n}^{\circ} 46=\mathrm{XIII}, 1,9107 ; \mathrm{n}^{\circ} 56=\mathrm{V}$, $\left.2212 ; \mathrm{n}^{\circ} 58=\mathrm{V}, 2665 ; \mathrm{n}^{\circ} 60=\mathrm{XVI}, 1,222\right)$. La plupart des autres inscriptions ont été publiées principalement dans Syria (textes $\left.n^{\circ s} 6,8,9,10,20,23,53,54,55,57\right)$ et dans les Annales archéologiques arabes syriennes (textes $\mathrm{n}^{\circ} 2,3,9,17,33,48,51$ ). Les autres inscriptions présentes dans le recueil sont extraites d'autres revues (Ktèma, 17, 1992, pour le texte $\mathrm{n}^{\circ} 22$; Zeitschrift für Papyrologie und Epigraphik, 47, 1982, pour le texte $\mathrm{n}^{\circ} 40$; Philologus 134, 1990 et 127, 1983, pour les textes $\mathrm{n}^{\text {os }} 41$ et 43 ; Damaszener Mitteilungen, 5, 1991 - revue absente de la liste des abréviations p. 217 - pour le texte $\mathrm{n}^{\circ} 50$; Tempora, 18, 2007, pour le texte $\mathrm{n}^{\circ} 53$; Annales d'histoire et d'archéologie, 8-9, 1997-1998, pour le texte $n^{\circ} 59$; Revue archéologique, 1884,pour le texte ${ }^{\circ} 62$; Travaux et Mémoires, 9,1985 , pour le texte $\mathrm{n}^{\circ} 64$ ). Certaines inscriptions sont également tirées de 
corpus épigraphiques (CIS, 3913 et $3971=$ textes $\mathrm{n}^{\text {os }} 4$ et 32 ; CIL, III, $133=\mathrm{n}^{\circ} 12$; Waddington, 2562 c, 1874-1875, 2512, 2545-2546, 2498, $\left.2036=\mathrm{n}^{\mathrm{os}} 15,30,44,47,49,61\right)$, des rapports des fouilles archéologiques de Doura Europos (Excavations, VI, 630 et V, 416 pour les textes $n^{\text {os }} 11$ et 35), de monographies (Y. Hajjar, La Triade d'Héliopolis-Baalbek, 1, p. 179-181 pour le texte $n^{\circ} 36$; C. Dunant, Le sanctuaire de Baalshamin, III, 25 pour le texte $\mathrm{n}^{\circ} 37$ ), enfin de colloques (Studia Aramaica, p. 34-37, pour le texte $\mathrm{n}^{\circ} 5$; Apamée de Syrie, p. 108-110, pour le texte $\mathrm{n}^{\circ} 29$; Société urbaines, sociétés rurales, p. 207-216 pour le texte $n^{\circ} 38$ ) et de mélanges (Mélanges Dussaud, p. 367-372, pour le texte $n^{\circ} 14$; Mélanges Collart, p. 31-46, pour le texte $\left.n^{\circ} 52\right)$.

5 Il convient enfin de mentionner trois inscriptions inédites (textes $n^{\text {os }} 26,31,63$ ) : un extrait en grec sur mosaïque d'une chronique d'Apamène au début du vi siècle; un milliaire en latin placé sur la route reliant Bostra à Adraha en 219 sous le règne d'Élagabal dont le nom a été martelé en raison de sa damnatio memoriae; une épitaphe chrétienne en grec datée de 397-398 mais d'origine inconnue car sauvée du trafic des antiquités. Cette liste un peu longue de références permet de suppléer l'absence de table de concordance, un appendice sans doute assez peu utile à un ouvrage de diffusion de la recherche.

6 La variété de ces travaux scientifiques se manifeste aussi par la richesse du détail et la précision remarquable des lemmes bibliographiques qui accompagnent chaque inscription présentée avec soin dans sa langue originale (l'araméen est translittéré en caractères latins), accompagnée d'une traduction privilégiant le sens à l'élégance, d'un commentaire concis et, en règle générale, d'une photographie quand le monument n'a pas disparu comme c'est le cas des textes $n^{o s} 11$ (dédicace de l'amphithéâtre militaire de Doura Europos) et 56 (épitaphe du tombeau pyramidal de Samsigéramos à Émèse). Pour de probables raisons de mise en page et de coût d'impression, les clichés sont d'une taille plutôt réduite, trop réduite pour permettre à l'occasion de lire des textes dont ne subsistent parfois que des traces évanescentes pour certaines pierres exposées aux outrages du temps comme les milliaires (voir les photographies assez peu lisibles accompagnant les textes $n^{\text {os }} 4 b$ et $c, 8,9,12,13,14 a, 15,16,21,27,31,32,33,34,37,39$, $41,42,50 \mathrm{~b}, 58,62)$. Dans quatre cas, les clichés sont accompagnés d'un dessin de la pierre qui en facilite beaucoup la lecture (textes $n^{\text {os }} 1,7,21 a$ et 25 concernant une ordonnance de Domitien à Épiphanée, un décret pour les pécheurs de pourpre d'Arados, une borne de Palmyrène et une épigramme latine sur un pressoir d'Apamène). Au risque d'accroître le nombre de pages et donc le prix du livre, cette solution aurait pu être facilement appliquée à toutes les inscriptions dont les photographies ne permettent guère la lecture.

7 Selon leurs spécialités et leurs inclinations, les sept auteurs ont proposé des témoignages épigraphiques de taille très variée, allant de quatre mots lisibles sur une dédicace au Zeus d'Héliopolis à de longs extraits du tarif de Palmyre (cf. textes $n^{\text {os }} 42$ et 4). La nature des inscriptions sélectionnées illustre également les principaux aspects - à l'exception des activités économiques dépourvues de rubrique particulière - de l'histoire antique de la Syrie, depuis l'époque hellénistique et surtout romaine jusqu'à l'invasion arabe et la brève reconquête byzantine, aux $\mathrm{x}^{\mathrm{e}}-\mathrm{XI}^{\mathrm{e}}$ siècles. Sont ainsi abordées, d'après le plan adopté par les auteurs, l'administration et la vie municipale, l'organisation militaire et défensive, les campagnes, les routes, les religions traditionnelles, les évergésies chrétiennes et les inscriptions funéraires. Ce choix se révèle heureux car il permet de surmonter la masse considérable des épitaphes, textes 
de surcroît souvent brefs, pauvres en informations et redondants. Par la richesse de ses textes, l'acribie de leurs traductions, la précision de leurs commentaires, la variété et, il faut le souligner, la remarquable beauté de l'iconographie et de la cartographie, ce recueil constitue un bel instrument de travail pour les enseignants et leurs étudiants en histoire ancienne.

\section{AUTEURS}

\section{SYLVAIN DESTEPHEN}

Université Paris Ouest - Nanterre La Défense 